\title{
Behavior of bounded solutions of quasilinear elliptic equations on Riemannian manifolds
}

\author{
A.B.Ivanov \\ Let $M$ be a connected complete noncompact Rimannian manifold of dimension $n, K$ is a \\ compact subset of $M$. We study bounded in $M \backslash K$ solutions of the equation

$$
\Delta_{p} u=c(x)|u|^{p-2} u
$$ \\ where $c(x) \in L_{\infty, l o c}(M)$ is a nonnegative function, $p>1$ is a real number, and operator $\Delta_{p}$ \\ is defined by

$$
\Delta_{p} u=g^{-\frac{1}{2}} \nabla_{i}\left(g^{i j}|\nabla u|^{p-2} \nabla_{j} u\right)
$$

where $g^{i j}$ are contravariant components of the metric tensor, $g=\operatorname{det}\left\|g^{i j}\right\|$, and

$$
|\nabla u|=\left(g^{i j} \nabla_{i} u \nabla_{j} u\right)^{\frac{1}{2}} .
$$

Similar problems were studied in [1]-[5].

A function $u \in W_{p, l o c}^{1}(\Omega) \cap L_{\infty}(\Omega)$ is said to be a solution of (1) in $\Omega$ if

$$
-\int_{\Omega} g^{i j}|\nabla u|^{p-2} \nabla_{i} u \nabla_{j} \varphi d x=\int_{\Omega} c(x)|u|^{p-2} u \varphi d x
$$

for every $\varphi \in C_{0}^{\infty}(\Omega)$.

We say a function $u \in W_{p, l o c}^{1}(\Omega) \cap L_{\infty}(\Omega)$ to be $p$-harmonic in $\Omega$, if

$$
\Delta_{p} u=0
$$

in the above sense.

Riemannian manifold $M$ is said to be an $H_{p}$-manifold if, for all non-empty open sets $\omega \subset \Omega \subset M$ such that

$$
\gamma_{1} \leq \frac{\operatorname{diam}(\omega)}{\operatorname{diam}(\Omega)} \leq \gamma_{2}
$$

and

$$
\gamma_{1} \leq \frac{\operatorname{dist}(\omega, \partial \Omega)}{\operatorname{diam}(\omega)} \leq \gamma_{2}
$$


where $\operatorname{diam}(\omega), \operatorname{diam}(\Omega)$ are the diameters of the sets $\omega$ и $\Omega$ correspondingly, $\operatorname{dist}(\omega, \partial \Omega)$ is the distance between $\omega$ and $\partial \Omega, \gamma_{1}, \gamma_{2}>0$ are real numbers, and for every nonnegative function $u$ that is p-harmonic on $\Omega$, the following inequality holds:

$$
\sup _{\omega} u \leq h \inf _{\omega} u
$$

where the constant $h>0$ depends only on $\gamma_{1}$ and $\gamma_{2}$.

Let $A$ be a compact, and $B$ an open subsets of $M, A \subset B$. The $p$ - capacity of $A$ with respect to $B$ is defined by

$$
\operatorname{cap}(A, B)=\inf _{\varphi} \int_{B}|\nabla \varphi|^{p} d x,
$$

where the infinum is taken over all functions $\varphi \in C_{0}^{\infty}(B)$ identically equal to 1 in a neighborhood of $A$.

Fix an arbitrary point $O \in M$.

We denote by $B_{k}=\left\{x: \operatorname{dist}(O, x)<2^{k}\right\}$ the geodesic ball of radius $2^{k}$ with center at $O$. For every set $\Omega$ denote $Q_{k}=\overline{B_{k}} \backslash \Omega, D_{k}=B_{k+2} \backslash \overline{B_{k+1}}$ and $d_{k}=\overline{B_{k+3}} \backslash B_{k}$.

An open set $\Omega$ is said to be $p$ - massive if

$$
\sum_{k=0}^{\infty}\left(\frac{\operatorname{cap}\left(Q_{2 k}, B_{2 k+2}\right)}{\operatorname{cap}\left(\overline{B_{2 k+1}}, B_{2 k+2}\right)}\right)^{\frac{1}{p-1}}<\infty .
$$

The main result of this paper is the following theorem.

Theorem 1. Let $M$ be a $H_{p}$-manifold and $c(x)$ satisfy

$$
\sum_{k=0}^{\infty}\left(\int_{d_{k}} \frac{c(x)}{\operatorname{cap}\left(d_{k}, D_{k}\right)} d x\right)^{\frac{1}{p-1}}=\infty
$$

for any $p$ - massive set $\Omega$. Then any bounded solution of (11) in $M \backslash K$ tends to zero when $\operatorname{dist}(O, x) \rightarrow \infty$

\section{References}

[1] V.A.Kondrat'ev, E.M.Landis "On qualitative properties of solutions of a nonlinear equation of second order", MATH USSR SB, 1989, 63 (2), 337-350.

[2] A.A.Kon'kov "On the Properties of Solutions to Elliptic Inequalities with Nonlinearity in the Principal Part", (in Russian) Doklady Akademii Nauk, 2002, V. 383 (1), 15-19.

[3] O.A.Ladyzhenskaya, N.N.Ural'tseva "Linear and Quasilinear Elliptic Equations", (in Russian) Nauka, Moscow, 1964. Engl. transl. American Mathematical Society, Providence, Rhode Island, 1968. 
[4] E.M.Landis "The second order equations of elliptic and parabolic type", (in Russian) Nauka, Moscow, 1971. Engl. transl. Transl. of Mathematical Monographs 171, AMS publications, 1998

[5] Grigor'yan, A. "Analytic and geometric background of recurrence and non-explosion of the Brownian motion on Riemannian manifolds", Bulletin of Amer. Math. Soc. 36 (1999), $135-249$ 